\title{
LA CREACIÓN DE UNA HERRAMIENTA COMPETENCIAL PARA ANALIZAR ACTIVIDADES BASADAS EN EL MLEARNING EN LA EDUCACIÓN PRIMARIA
}

\section{THE CREATION OF A COMPETENCIAL TOOL TO ANALYZE MLEARNING ACTIVITIES IN PRIMARY EDUCATION}

\author{
Balbina Falceto Macarulla \\ bfalceto@xtec.cat \\ Jordi Lluís Coiduras Rodríguez \\ coiduras@pip.udl.cat \\ Georgina Rovira Sanz \\ grovira6@xtec.cat
}

\begin{abstract}
Universidad de Lleida. Facultad de Educación, Psicología y Trabajo Social. Departamento de Pedagogía y Psicología. c/ Av. de l'Estudi General, 4, 25001, Lleida (España)
\end{abstract}

En este texto se expone el proceso de construcción de una herramienta de análisis de actividades con el uso de dispositivos móviles en Educación Primaria. El principal objetivo es seleccionar, definir y clasificar indicadores competenciales que permitan el estudio de las actividades con aplicaciones. La metodología seguida es mixta, combinando procesos cualitativos y cuantitativos. La búsqueda de referentes teóricos sobre tecnología educativa y competencias, la observación participante en un contexto con alumnos y la aportación del juicio de expertos permiten la creación de una herramienta para analizar el diseño de la actividad, la actuación de los alumnos y sus producciones.

Palabras clave: Análisis, competencia, herramienta, tecnología.

The research is based on the creation of a tool for competence analysis of activities which use mobile devices in Primary Education. The main objective is selecting, defining and classifying competence indicators for the activity analysis. The methodology to get this aim is based on qualitative and quantitative methods. The search for theoretical framework on educational technology and skills, combined with participant observation in a context with students and the contributions of expert's opinion, allows the creation of a tool to analyze the design of the activity, the performance of students and their productions.

Keywords: Analysis, competence, tool, technology.

Píxel-Bit. Revista de Medios y Educación. No 48 Enero 2016. ISSN: 1133-8482. e-ISSN: 2171-7966. doi: http://dx.doi.org/10.12795/pixelbit.2015.i48.02 


\section{Introducción.}

La principal motivación de este estudio fue desarrollar una herramienta de análisis de actividades en que se utilizan dispositivos móviles como herramienta de aprendizaje en la Educación Primaria, que permitiera conocer la relación del uso de dispositivos móviles con el desarrollo de competencias. La ausencia de literatura y de instrumentos para este análisis, desde el diseño de estos recursos hasta las producciones del alumnado en su uso, están en el origen de la investigación. Este estudio se realiza combinando la comprensión de las actividades desde un plano teórico y en la experimentación en el aula en un centro de Educación Infantil y Primaria que lleva a cabo actividades con el uso de tablets. Actualmente, las nuevas generaciones aprenden, se comunican y se divierten usando con frecuencia y facilidad las tecnologías, las cuales están muy presentes en sus tiempos. Igualmente, en el ámbito educativo el desarrollo de competencias es uno de los temas más actuales y hacia donde deben encaminarse los procesos educativos. Por esta razón, la importancia de conocer las posibilidades que las tecnologías, los dispositivos móviles en este caso, pueden aportarnos educativamente es otra de la motivación principal para la creación de esta herramienta. A la vez, esta motivación constituye en sí misma una finalidad de estudio, ya que como educadores e investigadores debemos disponer de herramientas que nos permitan analizar la funcionalidad educativa de las tecnologías.

Los dispositivos móviles representan la evolución continua de las tecnologías de la información y comunicación, también como una de las más recientes incorporaciones en los recursos escolares para aprender con tecnologías. Este desarrollo está modificando ya el trabajo en el aula, substituyendo no solamente papel y bolígrafo sino como nuevas prácticas en el acercamiento y trabajo de los contenidos, mediante usos y dinámicas pedagógicas nuevos. Para Moya (2013), las TIC a nuestra disposición pueden suponer mejoras en nuestra calidad de vida para gestionar la información: recibir, emitir, procesar, almacenar, recuperar y manipular. En esta dirección se han desarrollado cambios en la manera de concebir las nuevas tecnologías. Para Vivancos (2008), ya no se enseña sobre las TIC (Tecnologías de la Información y la Comunicación), sino que se aprende con y a través de la tecnología, con las TAC (Tecnología del Aprendizaje y el Conocimiento). Fornell y Vicancos (2010) definen las TAC como el uso de las tecnologías para una mejora en los procesos de enseñanza-aprendizaje, de evaluación y de organización, como agentes de cambios metodológicos que permitirán prácticas innovadoras y significativas.

Las tecnologías proponen progresivamente nuevos usos, dan respuesta a tareas de siempre con soluciones distintas o proponen nuevas utilidades para mejorar la información o la eficiencia en su gestión, además de promocionar en su uso una orientación autodidacta. Pedró (2006) apunta que el desarrollo de las competencias intelectuales y las capacidades cognitivas de las nuevas generaciones en un entorno digital transforma la forma de procesar y pensar. Los centros escolares deben dar respuesta a las necesidades que de estos cambios se derivan. De algún modo, en base a Gisbert \& Esteve (2011), los aprendices actuales comparten características comunes en cuanto a la forma de aprender e interactuar, en esta era digital: 
acceden a la información a partir de fuentes no impresas; prefieren las imágenes en movimiento y la música al texto; se sienten bien y cómodos realizando múltiples tareas al mismo tiempo; construyen conocimiento procesando información de forma discontinua y no lineal.

En un currículo por competencias, la significación y funcionalidad de los aprendizajes requiere nuevos modos de aprender y enseñar. La manera tradicional que ha imperado durante siglos en la escuela hoy en día ya no es suficiente, pudiendo identificarla con una «pedagogía tóxica» (Acaso, 2009), la cual genera desmotivación y fracaso escolar y también un desgaste en los docentes. De forma contraria, Trujillo (2012) afirma que se necesita una «pedagogía orgánica», que se ponga al servicio de los estudiantes para ayudarles a desarrollar competencias a través de tres ideas fundamentales: revisión curricular para garantizar los objetivos y su pertinencia, el interés de los contenidos y el valor de las prácticas de aprendizaje desde la visión del alumnado; apuesta por metodologías activas y dialógicas, con comunicación y descubrimiento compartido y adopción de estrategias y mecanismos que aseguren una evaluación para el aprendizaje dinámica y procesual.

En general, Marqués (1998) afirma que se necesita motivación para desarrollar competencias y lo define como un estado dinámico del alumno que lo ayude a comenzar una actividad y a ser constante durante su realización. En este sentido, para Navarro, Rigo y Roselló (2009) la competencia es la capacidad de utilizar de forma integrada todos los conocimientos, capacidades, actitudes y valores para poder superar con éxito diferentes tareas en un contexto determinado.

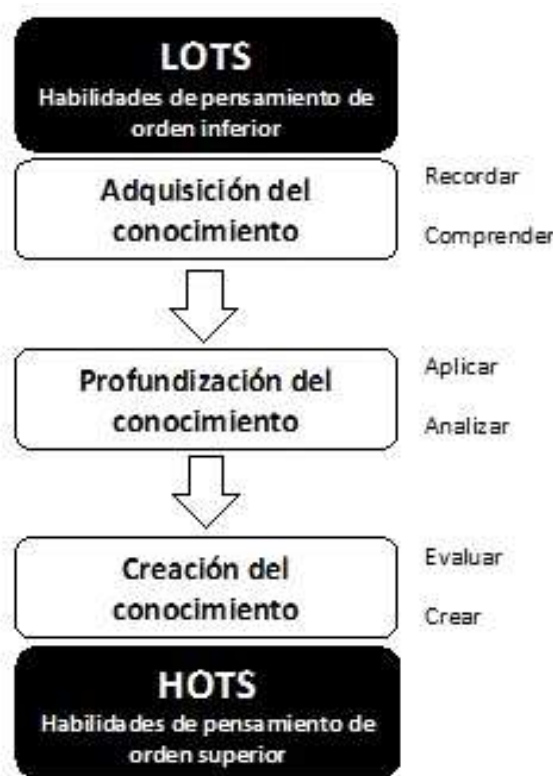

Figura 1. De la adquisición del conocimiento a la creación de éste (Churches, 2009).

De la misma manera, Scallon (2004) señala que se puede hablar de competencia cuando una persona es capaz de movilizar adecuadamente sus aprendizajes y el saber hacer en situaciones diferentes. La esencia de la competencia reside en la movilización de recursos que realiza el individuo, tanto los propios como los otros que le son externos para resolver una situación del contexto real (Le Boterf, 2002). Hoy en día muchos de estos recursos deben ser gestionados mediante medios tecnológicos, lo que hace que la competencia digital adquiera también una trascendencia crucial en la enseñanza y el aprendizaje. En base a Churches (2009), la educación del siglo XXI, con las nuevas tecnologías, está enfocada a ayudar a los alumnos a pasar de las habilidades de pensamiento de orden inferior a las de orden 
superior de la Taxonomía de Bloom (1956), para pasar de receptores, a creadores de conocimiento (Figura 1).

En la actualidad muchos de estos recursos abarcan o incorporan el componente tecnológico y, progresivamente, mediante tecnología móvil parte de la gestión de los procesos de resolución de las tareas. Inevitablemente, la tecnología móvil se introduce en los centros escolares, y con ella nuevos retos docentes para su selección en base a su adecuación a los objetivos educativos y metodologías para el desarrollo de las competencias.

Según Gisbert, Prats y Cabrera (2015), las tecnologías en general y los dispositivos móviles están en alza no sólo en los mercados, sino también desde otros puntos de vista: personal, social y académico. Los principales dispositivos móviles son los teléfonos, los reproductores de sonido, las consolas de videojuegos y las tabletas. Éstos están dentro de la tipología de aprendizaje del mLearning (del inglés Mobile Learning). Como señala la UNESCO (2013, p.6) «el aprendizaje móvil comporta la utilización de tecnología móvil, sola o en combinación con cualquier otro tipo de tecnología de la información y las comunicaciones (TIC), a fin de facilitar el aprendizaje en cualquier momento y lugar».

El mLearning viene imponiéndose en las distintas esferas de la actividad social, no sólo por las ventajas técnicas vinculadas a su portabilidad, adaptabilidad, inmediatez, simplicidad y rapidez (Nakano et al., 2013), sino también porque ofrece una serie de beneficios pedagógicos orientados al aprendizaje activo, situado, colaborativo, flexible, espontáneo e informal (Ally, 2012). Este tipo de aprendizaje es el que fomenta el desarrollo de competencias.
Existen investigaciones que han analizado la funcionalidad del uso de dispositivos móviles en la educación y que pueden ser útiles para el diseño de una herramienta que analice actividades centradas en el uso de tecnología. La investigación de Hoover y Valencia (2011) tuvo como objetivo determinar, a través de un caso experimental, los beneficios del uso del iPad en los resultados académicos de los alumnos. En general, se pudo observar que los alumnos que habían utilizado tablets durante el semestre académico obtuvieron puntuaciones más altas que aquellos otros que no lo utilizaron. En el trabajo de Fabian y MacLean (2014) también se presentó un estudio del uso de tablets en el ámbito educativo, con la metodología y los resultados de un proyecto de investigación de intervención / acción para evaluar los beneficios y riesgos potenciales del uso de dispositivos móviles en las actividades de enseñanza y aprendizaje en un entorno de educación superior. Según los resultados de su investigación, en términos de contenido de aprendizaje, se utilizaron tablets para: consumo de contenidos, creación de contenidos e intercambio de contenidos. En el diseño de las actividades llevadas a cabo en este proyecto piloto destacó el aprendizaje colaborativo, el discurso de los estudiantes y el apoyo entre iguales. Sin embargo, hay que apuntar que había tareas que no encajaban muy bien con el uso de tablets y había tareas que no eran adecuadas para llevar a cabo las actividades de colaboración. Finalmente, I \& J Management Services (2011) también realizaron un proyecto para evaluar las potencialidades de los $i \mathrm{Pad}$ para aprender. Según este estudio, la utilización del iPad en clase permite: 
Aumentar el aprendizaje independiente y autónomo entre los estudiantes.

Aumentar la motivación de los estudiantes y la participación activa en el aprendizaje.

Mejorar la capacidad de los profesores para planificar y satisfacer las necesidades individuales de los estudiantes.

Mejorar los resultados de aprendizaje de los estudiantes.

Extender el aprendizaje de los estudiantes más allá del aula.

Mejorar la participación de los padres en el aprendizaje y fortalecer los vínculos hogar-escuela.

En general, se puede concluir que estos estudios son favorables y el uso adecuado y educativo de tecnología permite mejorar los resultados de aprendizaje de los alumnos. Del mismo modo, Marés (2012) afirma que las tablets representan un nuevo formato de consumo y producción de contenidos y nuevas formas de interactividad, interacción y entretenimiento.

En el desarrollo de esta investigación, las experiencias escolares con tablets y aplicaciones de distribución gratuita son la base de la creación de una herramienta de análisis de aplicaciones y actividades pensadas para tecnología móvil.

\section{Metodología.}

La investigación se circunscribe en el paradigma interpretativo, acercándonos a la realidad desde metodologías fundamentalmente cualitativas, complementadas con algunos datos cuantitativos, y con la participación de los investigadores, como docentes, en el aula de Educación Primaria. Las prácticas educativas tienen lugar en un centro de Educación Primaria en Lleida en el área de inglés, donde se utiliza tecnología móvil, durante el curso 2014/15. A partir de un estudio de caso sobre las actividades que el alumnado realiza con aplicaciones en las tablets conocemos la propuesta educativa de la incorporación de esta tecnología en una metodología educativa de trabajo por rincones. Desde el análisis de la bibliografía construimos la herramienta de análisis, que validamos posteriormente por jueces y que sometemos a prueba mediante la observación y el análisis de las actividades que realizan los alumnos en 54 sesiones de clase.

A pesar de la existencia de herramientas e indicadores para el análisis de actividades, se prefiere diseñar una nueva con un enfoque competencial. Para ello se siguen dos procesos: el primero desde la observación de la práctica, inductivo, y posteriormente desde la revisión de la literatura, deductivo. La observación previa de la actividad del alumnado en el aula nos permite conocer la variedad de actividades que se utilizan en este caso en la enseñanza del inglés y la movilización que realiza el alumnado de habilidades y recursos y las interacciones que se presentan en el aula en esta situación.

Por consiguiente, las fases que se siguen para la creación de la herramienta son las siguientes:

a) Selección y definición de indicadores desde la observación en el aula.

b) Selección y definición de indicadores de fuentes teóricas relacionadas especialmente con la tecnología móvil y el desarrollo de las competencias.

c) Selección de los indicadores desde criterios de importancia, pertinencia, observabilidad y univocidad. 


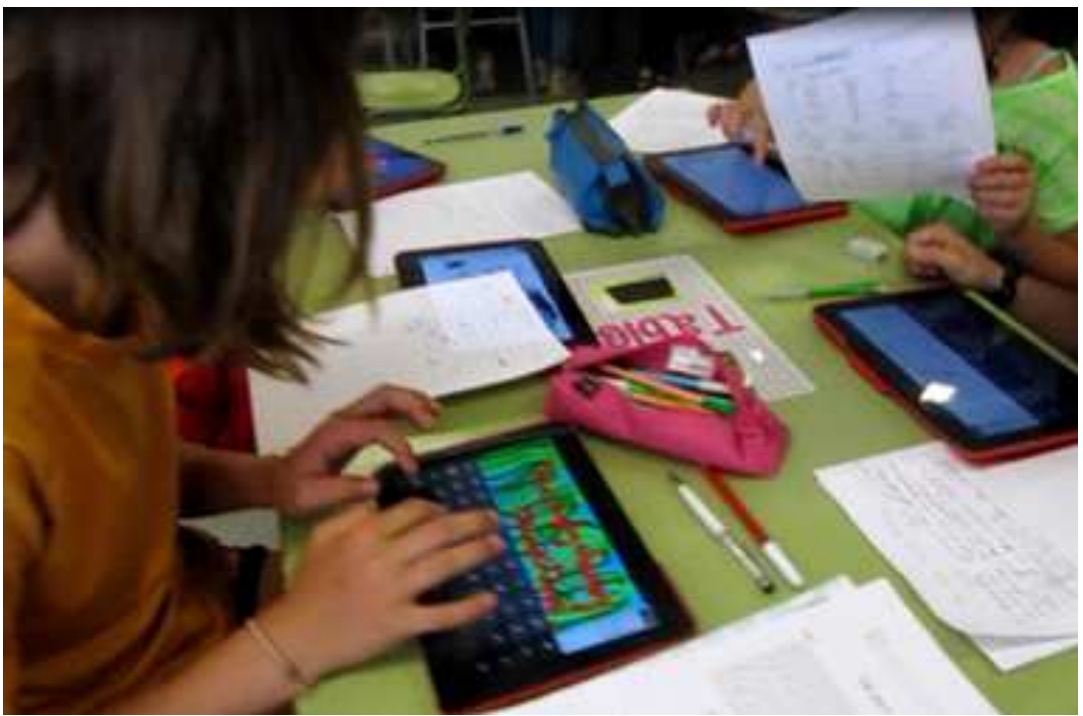

Imagen 1. Alumnado trabajando en el «rincón de la tablet».

d) Clasificación de los indicadores en dimensiones y categorías, en relación a: diseño de la actividad, actuación del alumnado y producciones del alumnado.

e) Definición de las dimensiones, categorías e indicadores.

f) Creación de un cuestionario online para la validación de contenido por expertos.

g) Revisión y modificación de la herramienta en base a las aportaciones y las puntuaciones del juicio de expertos y la realización de pruebas de análisis competencial con las actividades observadas en el contexto escolar.

Los principales referentes teóricos utilizados para la confección de la herramienta de análisis competencial de actividades con tablets son los siguientes:

Churches, A. (2008). Welcome to the 21st Century: Bloom's digital Taxonomy. Este autor presenta las habilidades cognitivas inferiores y superiores de la Taxonomía de Bloom (1956) complementadas con verbos digitales. Estos verbos (publicar, marcar, realizar búsquedas, etc.) permiten identificar el tipo de habilidad que se desarrolla en determinadas actividades con el uso de las tecnologías.

Decreto 142/2007, de 26 de junio, por el que se establece la ordenación de las enseñanzas de la Educación Primaria». Diario Oficial de la Generalitat de Catalunya (29 de junio de 2007). En este decreto se presentan las competencias básicas generales que el alumnado tiene que desarrollar en la Educación Primaria.

Gardner, H. (2011). Inteligencias múltiples: la teoría en la práctica. Este autor ideó la Teoría de las Inteligencias múltiples. Gardner (2011) reconoce que las personas son diferentes y tienen diferentes capacidades de pensar y diferentes formas de aprender.

Guilford, J.P. (1950). Creativity. American Psychologist. Guilford (1950) apuesta por el pensamiento divergente como el capaz de desarrollar producciones 


\begin{tabular}{|c|c|c|}
\hline DIMENSIÓN & CATEGORIA & INDICADOR \\
\hline \multirow{4}{*}{ Diseño de la actividad } & 1.Contextualización de la actividad & $\begin{array}{l}\text { 1.1.Relación con el entorno } \\
\text { 1.2.Promoción de una situación real }\end{array}$ \\
\hline & $\begin{array}{l}\text { 2. Globalidad de contenidos, competencias } \\
\text { y habilidades }\end{array}$ & $\begin{array}{l}\text { 2.1.Contenidos de diferentes ámbitos } \\
\text { temáticos } \\
\text { 2.2.Inteligencias múltiples } \\
\text { 2.3.Competencias básicas } \\
\text { 2.4.Habilidades de la era digital } \\
\text { 2.5.Codificación y/o descodificación en } \\
\text { diferentes formatos }\end{array}$ \\
\hline & 3.Utilización de materiales & $\begin{array}{l}\text { 3.1.Materiales y recursos necesarios } \\
\text { 3.2.Uso de recursos }\end{array}$ \\
\hline & 4.Agrupamiento del alumnado & 4.1.Modalidad de trabajo \\
\hline \multirow[b]{2}{*}{$\begin{array}{l}\text { Actuación del } \\
\text { alumnado }\end{array}$} & 5.Realización de la actividad & $\begin{array}{l}\text { 5.1.Autonomía } \\
\text { 5.2.Participación } \\
\text { 5.3.Interacción }\end{array}$ \\
\hline & ‘. Motivación & $\begin{array}{l}\text { 6.1.Decisión por comenzar la actividad } \\
\text { 6.2. Perseverancia en la realización de la } \\
\text { actividad } \\
\text { 6.3. Atención en la realización de la } \\
\text { actividad }\end{array}$ \\
\hline \multirow{2}{*}{$\begin{array}{l}\text { Producción del } \\
\text { alumnado }\end{array}$} & 7.Adecuación de los resultados & $\begin{array}{l}\text { 7.1.Presentación } \\
\text { 7.2.Corrección }\end{array}$ \\
\hline & 8.Creatividad & $\begin{array}{l}\text { 8.1.Originalidad } \\
\text { 8.2.Elaboración }\end{array}$ \\
\hline
\end{tabular}

Tabla 1. Dimensiones, categorías e indicadores de la herramienta creada y validada.

creativas, las cuales son analizadas desde seis dimensiones: fluidez (capacidad para dar diferentes respuestas ante un problema, elaborando más soluciones, más alternativas), flexibilidad (capacidad de cambiar de perspectiva, adaptarse a nuevas reglas, ver un mismo problema desde diferentes ángulos), originalidad (se refiere a la novedad desde un punto estadístico), redefinición (capacidad para buscar funciones y aplicaciones distintas a las habituales, para agilizar la mente), penetración (capacidad de profundizar, ir más allá) y elaboración (capacidad de incluir detalles).

. Puentedura, R. (2008). TPCK and SAMR: Models for enhancing technology integration. Otro autor que nos habla de la integración de las tecnologías en el currículo educativo es Puentedura (2008) con su 
modelo de Sustitución, Aumento, Modificación y Redefinición (SAMR, según su nomenclatura inglesa). La Sustitución se produce cuando se emplean las nuevas tecnologías como sustitutas directas de los métodos tradicionales. En cambio, se llega a la Redefinición cuando las nuevas tecnologías nos sirven para crear producciones antes inconcebibles.

La validación por expertos se realiza mediante dos vías: la Plataforma del Panel Internacional de Investigación en Tecnología Educativa, que ofrece a la comunidad académica un servicio de jueces expertos, evaluadores, seleccionados aleatoriamente, por su adecuación, especialidad y experiencia y la Universidad de Lleida, que dispone de profesionales entendidos en Tecnología Educativa. Los expertos validan la herramienta en un formulario en una escala de Likert (1-4), valorando su importancia, pertinencia, observabilidad y univocidad: importancia como trascendencia del ítem para el objeto de estudio; pertinencia como grado de adecuación del ítem al criterio dentro del cual se encuentra; observabilidad como calidad del ítem en relación a la posibilidad de ser observado y, por último, univocidad, como medida de como el ítem puede ser comprendido o interpretado inequívocamente de una única forma. Cuando se reciben las respuestas de los expertos, la información se analiza de forma cuantitativa y cualitativa para modificar la herramienta de análisis cuando hay convergencia en las aportaciones valorativas o los comentarios abiertos proponen mejoras en la comprensión del texto.

La muestra de alumnos seleccionados para observar en su contexto escolar son tres grupos de Educación Primaria, de cuarto, quinto y sexto curso. La observación participante tiene lugar en la clase de inglés y precisamente con este alumnado porque son los que más tiempo llevan trabajando por metodología de rincones, en grupos de cuatro o cinco y usando la tecnología móvil. El «rincón de la tablet» es el rincón donde los alumnos realizan actividades con el uso de aplicaciones. En cada clase hay entre 20 y 25 alumnos. En la Imagen 1 se puede observar dicho rincón con alumnado trabajando:

\section{Resultados.}

Después de la validación de expertos y sus aportaciones y del pilotaje de la herramienta, esta queda definida como mostramos en la Tabla 1, con dimensiones, categorías e indicadores.

En la consideración de las puntuaciones aportadas por los expertos en relación al ítem interesa la observabilidad en primer lugar, para poder objetivar la valoración y la importancia, como trascendencia en el análisis y valoración. La pertinencia y univocidad valoradas nos permiten tener en cuenta el ítem, mantenerlo, o revisar la redacción para asegurar la comprensión en un sentido único.

Para entender el funcionamiento de la herramienta, se muestran a modo de ejemplo los resultados del análisis de una actividad en la Tabla 2. En primer lugar, se presenta la ficha de presentación de la actividad para entender su fundamentación y la producción de un alumno (véase Imagen 2).

FICHADEANÁLISIS:

Actividad no: 3

TÍTULO:

(Comparativos)

Comparatives

CURSO: $4^{\circ}$ Primaria

APLICACIÓN: Skitch

TIPO DE RECURSO: Imagen 
LINK: https://play.google.com/store/ apps/details?id=com.evernote. skitch\&hl=es COMPETENCIAS:

Competencia comunicativa lingüística y audiovisual

Competencia artística y cultural

Tratamiento de la información y competencia digital

Competencia matemática

Competencia de aprender a aprender

Competencia de autonomía e iniciativa personal

Competencia en el conocimiento y la interacción con el mundo físico

\section{- DESCRIPCIÓN:}

La aplicación utilizada en esta actividad, Skitch, sirve para editar imágenes. En este caso los alumnos de cuarto trabajan el concepto gramatical de comparativos. Deben ir por la escuela con la tablet y hacer una fotografía de dos cosas diferentes y luego con la aplicación Skitch escribir la frase con comparativos. Pueden emplear todas las opciones que la aplicación permite: hacer cuadros, flechas, rodear, etc. Posteriormente, comparan animales y también se comparan entre ellos mismos.

MODALIDAD DE TRABAJO: Individual, cada alumno con su tablet

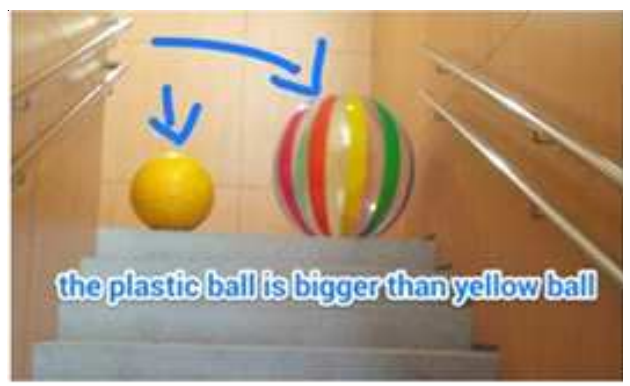

Imagen 2. Producción real de un alumno.
Con la herramienta, obtenemos el análisis que mostramos en la Tabla 2. Algunos de los indicadores tienen asignada una puntuación, entre 1, la mínima, y 5, la máxima. Otros indicadores son de opción múltiple para seleccionar (inteligencias múltiples, competencias, etc.).

La observación de las actividades en el aula es un modo de pilotaje, de verificación del funcionamiento de la herramienta en su formato, contenido y funcionalidad para estudiar la vinculación de la tecnología móvil y el desarrollo de competencias.

La validación mediante el juicio de expertos aporta algunos matices y propuestas, que modifican y reestructuran en parte la herramienta. En la Tabla 3 se puede observar el sumatorio de valores en las respuestas que los expertos han otorgado en la escala a cada valor.

El juicio de los expertos valida en general los indicadores propuestos con el mayor número de respuestas en el valor 4. En las aportaciones textuales, los expertos proponen algunos cambios en la redacción de los indicadores y el replanteamiento de algunos que presentan dificultades en su condición de observabilidad, como el nivel de esfuerzo cognitivo, los conocimientos previos del alumno, etc. En caso de puntuaciones inferiores a 3 se modifican o eliminan. En el proceso de revisión y modificación de la herramienta, nos hemos planteado cuestiones como las que se presentan a continuación:

¿Cómo sabemos que un alumno desarrolla competencias? En este sentido, el indicador referente a las competencias se pasa a la dimensión de «Diseño de la actividad». Cuando planificamos la actividad, pensamos sobre las competencias que 


\begin{tabular}{|c|c|c|}
\hline \multicolumn{3}{|c|}{ ACTIVIDAD: COMPARATIVES (COMPARATIVOS) } \\
\hline DIMENSIÓN & CATEGORÍA & INDICADOR \\
\hline \multirow{4}{*}{$\begin{array}{l}\text { Diseño de la } \\
\text { actividad }\end{array}$} & $\begin{array}{l}\text { 1.Contextualización de la } \\
\text { actividad }\end{array}$ & $\begin{array}{l}\text { 1.1. Relación con el entorno. Puntuación: } 5 \\
\text { 1.2. Promoción de una situación real. Puntuación: } 5\end{array}$ \\
\hline & $\begin{array}{l}\text { 2.Globalidad de } \\
\text { contenidos, competencias } \\
\text { y habilidades }\end{array}$ & $\begin{array}{l}\text { 2.1. Contenidos de diferentes ámbitos temáticos. Puntuación: } 4 \\
\text { 2.2. Inteligencias múltiples: Inteligencia lingüística, Inteligencia } \\
\text { lógico-matemática, Inteligencia visual-espacial, Inteligencia } \\
\text { cinético-corporal, Inteligencia interpersonal, Inteligencia } \\
\text { intrapersonal. } \\
\text { 2.3. Competencias básicas: Competencia comunicativa } \\
\text { lingüística y audiovisual, Competencia artística y cultural, } \\
\text { Tratamiento de la información y competencia digital, } \\
\text { Competencia matemática, Competencia de aprender a aprender, } \\
\text { Autonomía e iniciativa personal, Competencia en el } \\
\text { conocimiento y la interacción con el mundo físico } \\
\text { 2.4. Habilidades de la era digital: Crear } \\
\text { 2.5. Codificación y/o descodificación en diferentes formatos: } \\
\text { Texto, Imagen. }\end{array}$ \\
\hline & 3.Utilización de materiales & $\begin{array}{l}\text { 3.1. Materiales y recursos necesarios: Aplicaciones de la tableta } \\
\text { digital, Enunciado, modelo o guía de la actividad, Otras } \\
\text { herramientas de la tableta: cámara, captura de pantalla, } \\
\text { calculadora, nota de voz,... } \\
\text { 3.2. Uso de recursos: Uso de recursos como redefinición. }\end{array}$ \\
\hline & $\begin{array}{l}\text { 4.Agrupamiento del } \\
\text { alumnado }\end{array}$ & $\begin{array}{l}\text { 4.1. Modalidad de trabajo: Otros: alternancia trabajo individual } \\
\text { y trabajo en grupo, por parejas... }\end{array}$ \\
\hline \multirow[b]{2}{*}{$\begin{array}{l}\text { Actuación del } \\
\text { alumnado }\end{array}$} & $\begin{array}{l}\text { 5.Realización de la } \\
\text { actividad }\end{array}$ & $\begin{array}{l}\text { 5.1. Autonomía: Todos los alumnos se muestran autónomos } \\
\text { 5.2. Participación: Todos los alumnos participan } \\
\text { 5.3. Interacción: Todos los alumnos interactúan }\end{array}$ \\
\hline & 6. Motivación & $\begin{array}{l}\text { 6.1. Decisión por comenzar la actividad: Todos los alumnos } \\
\text { muestran decisión. } \\
\text { 6.2. Perseverancia en la realización de la actividad: Algunos } \\
\text { alumnos se muestran perseverantes. } \\
\text { 6.3. Atención en la realización de la actividad: Algunos } \\
\text { alumnos se muestran atentos. }\end{array}$ \\
\hline \multirow{2}{*}{$\begin{array}{l}\text { Producción del } \\
\text { alumnado }\end{array}$} & $\begin{array}{l}\text { 7.Adecuación de los } \\
\text { resultados }\end{array}$ & $\begin{array}{l}\text { 7.1. Presentación. Puntuación: } 5 \\
\text { 7.2. Corrección. Puntuación: } 5\end{array}$ \\
\hline & 8.Creatividad & $\begin{array}{l}\text { 8.1. Originalidad: Puntuación: } 5 \\
\text { 8.2. Elaboración: Puntuación: } 4\end{array}$ \\
\hline
\end{tabular}

Tabla 2. Análisis de una actividad con la herramienta de investigación diseñada. 


\begin{tabular}{|c|c|}
\hline VALOR & SUMATORIO POR VALORES \\
\hline 1 & 46 \\
\hline 2 & 177 \\
\hline 3 & 290 \\
\hline 4 & 415 \\
\hline
\end{tabular}

Tabla 3. Sumatoria de respuestas de los expertos.

queremos que los alumnos desarrollen a través de las tareas.

$¿$ La fluidez puede observarse en las actuaciones de los alumnos y sus producciones? Es mucho más claro comparar la originalidad o el nivel de producción de cualquier actividad, que la fluidez del alumnado al pensar y realizar actividades.

\section{Discusión.}

La observación participante y las valoraciones del juicio de expertos han permitido la modificación y desarrollo de la herramienta creada, para adaptarla con mayor exactitud al tipo de medio educativo utilizado en las actividades, tablets, y a los requisitos educativos que definen un proceso de enseñanza-aprendizaje con enfoque competencial.

Finalmente, se compone de tres dimensiones (ver Tabla 1): diseño de la actividad, actuación del alumnado y producción del alumnado. Las tres mantienen cierta correspondencia con las tres ideas fundamentales que nos proponía Trujillo (2012), con su «pedagogía orgánica»: la revisión curricular de los objetivos estaría situada en el «Diseño de la actividad»; el interés de los contenidos y el valor de las prácticas desde la percepción del alumnado se analizaría a través de la «Actuación del alumnado» y finalmente, en la evaluación de estas prácticas para corroborar su grado de funcionalidad se tendría en cuenta la «Producción del alumnado».

Si nos centramos en la dimensión «Diseño de la actividad» de la herramienta, los indicadores más relevantes para el análisis de las actividades y nuestro objeto de estudio se encuentran en la categoría referente a «Globalidad de contenidos, competencias y habilidades», ya que en ésta se engloban las competencias (Decreto 142/2007), las habilidades de orden cognitivo adaptadas a la era digital (Churches, 2009) y las inteligencias múltiples de Gardner (2011) consideradas o que pueden reconocerse entre las potencialidades de la propuesta o diseño de las actividades con el uso de tablets, entre otros aspectos.

En referencia a la dimensión «Actuación del alumnado» de la herramienta diseñada, en las distintas sesiones de observación, se ha constatado que, como señalan Gisbert y Esteve (2011), los alumnos que aprenden utilizando tablets como medio educativo están especialmente motivados por las imágenes, los vídeos y la música. Dentro de la categoría referente a la motivación, se han considerado 
tres indicadores para el análisis: decisión para comenzar la actividad, perseverancia en la realización de la actividad y atención en la realización de la actividad. Estos indicadores nos acercan al concepto de motivación, tomando como referencia a Marqués (1998).

Finalmente, la dimensión «Producción del alumnado» se relaciona especialmente con la adecuación de los resultados y la creatividad. Como se constata en otras investigaciones relacionadas con el uso de tablets en la educación (Hoover \& Valencia, 2011; I \& J Management Services, 2011), los resultados apuntan que el uso de dispositivos móviles de forma educativa mejora los resultados de los estudiantes. Por este motivo, se ha considerado muy oportuno incluir en la herramienta una dimensión referente a la observación y el análisis de los resultados, a través de las producciones del alumnado. Lo que se observa y analiza a partir de estas producciones son, especialmente, algunos de los conceptos que Guilford (2011) tiene en cuenta en su apuesta por el pensamiento divergente en los alumnos, como la elaboración, la originalidad, etc. Algunos de los expertos que validan la herramienta consideran oportuno analizar si existe variedad de producciones y su grado de originalidad para determinar si un proceso educativo es significativo y adaptado a las necesidades del alumnado.

Este estudio da relevancia tanto al análisis como al diseño y a la evaluación de actividades mediante tablets. El diseño también que como docentes proponemos en el uso de la actividad, la actuación de los alumnos desarrollando tareas concretas y los resultados como producciones que estos generan.

Los tres conceptos determinan un análisis sencillo y a la vez amplio, desde el planteamiento hasta la obtención de resultados. La herramienta presentada es de interés docente porque permite un acercamiento a la actividad y a la aplicación estudiando las intenciones, los objetivos implícitos y las habilidades que entran en acción, y a la vez, contrastado con resultados concretos.

Como herramienta puede facilitar la reflexión sobre el tipo de actividades que planteamos o hacemos uso: ¿Son actividades de ejercitación para la mejora de habilidades o aprendizajes por repetición? ¿Tienen potencialidad constructivista porqué sitúan al alumno ante un reto que deberá resolver mediante la combinación de lo aprendido con aprendizajes nuevos? ¿Las actividades planteadas son diversas y consideran distintas inteligencias o por el contrario insisten en algunas de ellas y obvian otras? ¿La tecnología se usa como una simple substitución de los recursos para hacer las tareas de siempre o mediante ella el alumno aborda tareas nuevas, antes inconcebibles? La herramienta no tiene sentido por sí misma, solamente podemos considerarla una aportación si nos conduce a la reflexión sobre el aprendizaje, las actividades propuestas, así como un uso productivo y educativo de la tecnología.

Como línea de investigación futura, interesa plantearse la utilización de la herramienta en actividades que se basen en el uso de diferentes dispositivos tecnológicos. Esta acción permitiría poder realizar una comparativa de los resultados obtenidos, en función del medio educativo utilizado. Igualmente, sería conveniente pilotar la herramienta en distintos niveles educativos. 


\section{Referencias bibliográficas.}

Acaso, M. (2009). La educación artística no son manualidades. Madrid: Catarata.

Ally, M. (2012). Mobile Learning: The Equalizer in Education.LaEduc@ción Digital Magazine, 147, 1-13. Recuperado de h t t p : / / educoas.org/portal/ la_educacion_digital/147/pdf/ ART_Athabasca_EN.pdf

Bloom, S. (1956). Taxonomy of Educational Objectives, the classification of educational goals. Handbook I: Cognitive Domain. New York: McKay.

Churches, A. (2008). Welcome to the 21st Century: Bloom's digital Taxonomy. Educational Origami. Recuperado de http:// edoriga mi.wikis paces.com/ Bloom\%27s+Digital+Taxonomy

Churches, A. (2009). Bloom's digital taxonomy. Educational Origami. Recuperado de http:// edorigami.wikis paces.com/ Bloom\%27s+Digital+Taxonomy

«Decreto 142/2007, de 26 de junio, por el que se establece la ordenación de las enseñanzas de la Educación Primaria». Diario Oficial de la Generalitat de Catalunya (29 de junio de 2007), núm. 4915, págs. $21822-$ 21870.

Fabian, K. \& Maclean, D. (2014). Keep taking the tablets? Assessing the use of tablet devices in learning and teaching activities in the Further Education sector. Research in Learning Technology, 22, 1-14. doi: 10.3402/ rlt.v22.22648

Fornell, R. \& Vivancos, J. (2010). Educació el Pla TAC de centre: Col-lecció TAC-1. Servei de Tecnologies per a l'Aprenentatge i el Coneixement. Departament d'Educació. Generalitat de Catalunya. Recuperado de http://www.bcn.cat/imeb/pec/forum_exit/ Pla_Tac_de_centre.pdf

Gardner, H. (2011). Inteligencias múltiples: la teoría en la práctica. Barcelona: Paidós.

Gisbert, M. \& Esteve, F. (2011). Digital learners: La competencia digital de los estudiantes universitarios. La Cuestión Universitaria, 7, 48-59.

Gisbert, M., Prats, M. \& Cabrera, N. (2015). Aprenentatge móvil. Com incorporar els dispositius mòbils a l'aprenentatge?. Informes breus Educació, 58, 1-144. Barcelona: Fundació Jaume Bofill.

Guilford, J.P. (1950). Creativity. American Psychologist, 5 (9), 444-454.

Hoover, D. \& Valencia, J. (2011). iPads in the Classroom: Use, Learning Outcomes, and the Future. Presentation. Proceedings of the 2011 EDUCAUSE Annual Conference, Philadelphia, PA.

I \& J Management Services (2011). iPads for learning. In their hands trial. iPads for learning. Department of Education and Early Childhood Development, 1-66.

Le Boterf, G. (2002). Ingeniería de las Competencias. Barcelona: Gestion - Training Club-PISE.

Marés, L. (2012). Tablets en educación. Oportunidades y desafíos en políticas uno a uno. Relpe, Red latinoamericana portales educativos, 13. Recuperado de http:// www.oei.es/70cd/Tabletseneducacion.pdf

Marqués, P. (1998). Usos educativos de Internet. ¿La revolución de la enseñanza?. Recuperado de https://engage.intel.com/ servlet/JiveServlet/previewBody/24511-1021-29846/LaRevolucionDeLaEnsenanza.pdf

Moya, M. (2013). De las TICs a las TACs: la importancia de crear contenidos digitales educativos. Revista DIM, 27. Recuperado de http://dim.pangea.org/revistaDIM27/docs/ AR27contenidosdigitalesmonicamoya.pdf 
Nakano, T., Mija, A., Begazo, J., Garret, P., Velasco, A. \& Rosales, A. (2013). Uso de tablets en la educación superior: Una experiencia con iPads. Digital Education Review, 24, 135-167. Recuperado de http:// www.raco.cat/index.php/DER/article/view/ 271263/358905

Navarro, A., Rigo, C. \& Rosselló, R. (2009). Les competències bàsiques una resposta als reptes educatius. Palma: Conselleria d'Educació i Cultura.

Pedró, F. (2006). Aprender en el nuevo milenio: Un desafío a nuestra visión de las tecnologías y la enseñanza. Paris: OECDCERI. Recuperado de http:// i d b d o c s . i a d b. or g/w s d o c s / getdocument.aspx?docnum $=848274$

Puentedura, R. (2008). TPCK and SAMR: Models for enhancing technology integration. As we may teach: Educational technology, from theory into practice. Maine Department of Education.

Scallon, G. (2004). La evaluation des apprentisages dans une approche par competences. Québec: Ed. Du Renouveau Pédagogique.

Trujillo, F., (2012). Propuestas para una escuela en el siglo XXI. Madrid: Catarata.

UNESCO (2013). Policy guidelines for mobile learning. París: UNESCO.

Vivancos, J. (2008). Tratamiento de la información y competencia digital. Madrid: Alianza.

Fecha de recepción: 20-09-2015

Fecha de evaluación: 06-10-2015

Fecha de aceptación: 02-11-2015 